PROCEEDINGS OF THE

AMERICAN MATHEMATICAL SOCIETY

Volume 137, Number 10, October 2009, Pages 3425-3436

S 0002-9939(09)09900-6

Article electronically published on March 30, 2009

\title{
A LONG TIME ASYMPTOTIC BEHAVIOR OF THE FREE BOUNDARY FOR AN AMERICAN PUT
}

\author{
CHEONGHEE AHN, HI JUN CHOE, AND KIJUNG LEE
}

(Communicated by Walter Craig)

\begin{abstract}
In this paper we obtain a long time asymptotic behavior of the optimal exercise boundary for an American put option. This is done by analyzing an integral equation for the rescaled exercise boundary derived from the corresponding Black-Scholes partial differential equation with a free boundary.
\end{abstract}

\section{INTRODUCTION}

An option is a derivative security based on an underlying asset, for instance, a stock. We are interested in American put options (see 5]). The holder of an American put has the right to sell the underlying asset at any time prior to a fixed expiration date $T$. In this case she needs the function $\tilde{b}$ defined on the time up to $T$ to exercise her right as soon as the stock price falls below this function. We call $\tilde{b}$ the optimal exercise boundary. So far, the optimal exercise boundary and the corresponding option price in closed form with mathematical rigor haven't been found. However, some information about this boundary is available. See, for instance, [1], 2], 3], 4, and the references therein. In particular, the behavior of the boundary near the expiration time, the short time asymptotic behavior, has been studied intensively. But, we couldn't find the results related to the long time version in the literature. This motivated the present paper.

For further discussion of our results related to this unknown boundary we start with the following mathematical formulations. We assume that an underlying stock price is a log-normal process $S$ with the stochastic differential,

$$
d S_{t}=r S_{t} d t+\sigma S_{t} d B_{t},
$$

where $B$ is a one-dimensional Brownian motion defined on a complete probability space, the constants $r, \sigma$ represent the risk-free interest rate and the volatility of the stock, respectively. We also assume that the stock generates no dividends. Denoting the strike price by $K$, we let $F(\tau, S)$ denote the price of the American

Received by the editors April 30, 2008, and, in revised form, November 27, 2008, and January $27,2009$.

2000 Mathematics Subject Classification. Primary 91B28, 35R35; Secondary 45G05.

Key words and phrases. American put option, optimal exercise boundary, free boundary problem.

The second author is supported by the Korea Research Foundation Grant funded by the Korean Government (MOEHRD, Basic Research Promotion Fund) KRF-2007-314-C00020.

The third author is supported by BK21 project of Department of Mathematics in Yonsei University (R01-2004-000-10072-0) and settlement research fund by Ajou University.

(C)2009 American Mathematical Society Reverts to public domain 28 years from publication 
put option with the underlying stock price $S$ with the remaining time $\tau$ to the expiration. We define the optimal exercise boundary $b=b(\tau)$ on these remaining times $\tau$. The following observations are immediate: If the stock price with the remaining time $\tau$ becomes below $b(\tau)$, the holder of the option must sell the asset at the price $K$ right away. Hence, the reasonable price $F(\tau, S)$ of the option is $K-S$. Moreover, we can easily see that $b(0)$, the value of $b$ at the expiration, has to be $K$. This means that $F(0, S)=(K-S)_{+}$.

In fact, as a function on $\Omega_{F}:=[0, \infty) \times(0, \infty)$, the price of the American put $F(\tau, S)$ satisfies

$$
F(\tau, S)=K-S \text { for } \tau>0, S \leq b(\tau)
$$

and in the rest of the domain it solves the following second order parabolic partial differential equation with the free boundary $b$ crossing the domain $\Omega_{M}$ (cf. [11]):

$$
F_{\tau}-\frac{1}{2} \sigma^{2} S^{2} F_{S S}-r S F_{S}+r F=0 \quad \text { for } \quad \tau>0, S>b(\tau)
$$

with conditions

$$
\begin{gathered}
F(0, S)=(K-S)_{+}, \quad F(\tau, b(\tau))=K-b(\tau), \\
F_{S}(\tau, b(\tau))=-1, \quad \lim _{S \rightarrow \infty} F(\tau, S)=0 .
\end{gathered}
$$

One can couple this problem with its stationary version which finds the value $\bar{b}$ and the function $\bar{F}=\bar{F}(S)(S>0)$ satisfying

$$
\bar{F}(S)=K-S \quad \text { for } \quad 0<S \leq \bar{b}, \quad \frac{1}{2} \sigma^{2} S^{2} \bar{F}_{S S}+r S \bar{F}_{S}-r \bar{F}=0 \quad \text { for } \quad S>\bar{b}
$$

with conditions $F(\bar{b})=K-\bar{b}, \bar{F}_{S}(\bar{b})=-1, \lim _{S \rightarrow \infty} \bar{F}(S)=0$. This free boundary problem has the solution (see, for instance, 8])

$$
\bar{b}=K \cdot \frac{\rho}{\rho+1}, \quad \bar{F}(S)=(K-\bar{b})\left(\frac{S}{\bar{b}}\right)^{-\rho} \quad \text { for } \quad S>\bar{b}
$$

with $\rho:=\frac{2 r}{\sigma^{2}}$. The function $\bar{F}$ is called the perpetual put, or the Merton perpetual put; the value $\bar{b}$ is the optimal exercise boundary of it.

It is known that the boundary $b$ is convex (see [2, 3] for this nontrivial fact), smooth on $\tau \in[0, \infty) \backslash\{0\}$ (see [1]), and strictly decreases to $\bar{b}$ (see [7]). The option price $F$ is, of course, positive, and it is known (see [7] again) that $F$ is decreasing as $S$ goes to $\infty$, increasing as $\tau$ increases and that it converges to $\bar{F}$.

By, for instance, [1] our original problem (1.2) and (1.3) with corresponding conditions can be written in a simpler form by introducing new variables and functions defined by

$$
S=K e^{x}, \quad \tau=\frac{2}{\sigma^{2}} t, \quad \beta(t)=\ln \left(\frac{b(\tau)}{K}\right), \quad P(t, x)=\frac{1}{K} F(\tau, S) .
$$

In fact, $P$ defined on $\Omega_{P}:=[0, \infty) \times \mathbb{R}$, solves

$P_{t}-P_{x x}-(\rho-1) P_{x}+\rho P=0, t>0, x>\beta(t) ; \quad P(t, x)=1-e^{x}, t>0, x \leq \beta(t)$

with conditions

$P(0, x)=\left(1-e^{x}\right)_{+}, \quad P(t, \beta(t))=1-e^{\beta(t)}, \quad P_{x}(t, \beta(t))=-e^{\beta(t)}, \quad \lim _{x \rightarrow \infty} P(t, x)=0$. 
We note $\beta(t) \leq 0$ with $\beta(0)=0$. Similar to $b$, the free boundary $\beta(\cdot)$ crossing $\Omega_{P}$ is convex (see 2] again), smooth on $t \in[0, \infty) \backslash\{0\}$, and decreases to $\bar{\beta}:=\ln \left(\frac{\rho}{\rho+1}\right)$. The rescaled option price $P$ is positive, decreasing as $x$ goes to $\infty$, increasing as $t$ increases and it converges to

$$
\bar{P}(x)= \begin{cases}\frac{1}{1+\rho} e^{-\rho(x-\bar{\beta})} & \text { if } x>\bar{\beta} \\ 1-e^{x} & \text { if } x \leq \bar{\beta} .\end{cases}
$$

In this paper we study the convergence speed of $\beta(t), P(t, x)$ as they approach $\bar{\beta}, \bar{P}(x)$ respectively when $t \rightarrow \infty$. For $\beta$ we will make use of the following integral equation from [4] or [1]:

$$
G(t, \beta(t))=-\rho \int_{0}^{t} G(t-s, \beta(t)-\beta(s)) \beta^{\prime}(s) d s,
$$

where $G$ is the fundamental solution corresponding to the second order parabolic partial differential equation in (1.4), that is,

$$
G(s, y)=\frac{1}{\sqrt{4 \pi s}} \exp \left[-\frac{(y+(\rho-1) s)^{2}}{4 s}-\rho s\right], \quad s>0, y \in \mathbb{R} .
$$

For helping us understand where (1.7) comes from and for Theorem 1.2 below, we spend a paragraph for a derivation of it by paraphrasing what is written in [4] or [1. Note that we have a standard expression of $P$ using $G$ as a kernel:

$$
P(t, x)=(G(t, \cdot) * P(0, \cdot))(x)+\int_{0}^{t}\left(G(t-s, \cdot) * f_{P}(s, \cdot)\right)(x) d s,
$$

where $*$ means convolution with respect to the space variable $x$ and $f_{P}(s, x)=0$ for $x>\beta(s), f_{P}(s, x)=\rho$ for $x \leq \beta(s)$. We easily see that (1.8) is the same as

$$
P(t, x)=\int_{-\infty}^{0}\left(1-e^{y}\right) G(t, x-y) d y+\rho \int_{0}^{t} \int_{-\infty}^{\beta(s)} G(t-s, x-y) d y d s .
$$

By taking the derivatives of (1.9) with respect to $t$, using the fact that $G_{t}-G_{x x}$ $-(\rho-1) G_{x}+\rho G=0$, and integration by parts, one can derive

$$
P_{t}(t, x)=G(t, x)+\rho \int_{0}^{t} G(t-s, x-\beta(s)) \beta^{\prime}(s) d s, \quad x>\beta(t) .
$$

Also, we get

$$
P_{t}(t, \beta(t))=0
$$

by considering $\frac{d}{d t}(P(t, \beta(t)))$ and using (1.5). Now, equation (1.7) follows as we let $x \rightarrow \beta(t)^{+}$in (1.10).

We state our main theorems related to $\beta$ and $P$. The notation $o(\cdot)$ below means small oh.

Theorem 1.1. For any given $\rho>0$ we have

$$
\beta(t)=\bar{\beta}+o\left(e^{-\frac{(\rho+1)^{2}}{4} t}\right)
$$

as $t \rightarrow \infty$. 
Remark 1.1. An approximation of the long time behavior of $\beta$ is stated in Section 1 and proved in Section 3 and Subsection 7.7 of [1] It implies that $\beta(t)-\bar{\beta}$ can be approximated by some $O\left(t^{-\frac{3}{2}} e^{-\frac{(\rho+1)^{2}}{4} t}\right)$, which, in particular, is $o\left(t^{-\frac{3}{2}+\varepsilon} e^{-\frac{(\rho+1)^{2}}{4} t}\right)$ for any $\varepsilon>0$. But, according to the argument in the second paragraph of Subsection 7.7, this approximation is mathematically obscure, and this fact prevents us from concluding that $\beta(t)-\bar{\beta}$ is $O\left(t^{-\frac{3}{2}} e^{-\frac{(\rho+1)^{2}}{4} t}\right)$. Meanwhile, our expression in Theorem 1.1 is mathematically safe, and the proof is quite elementary.

Applying the formula (1.8) to $h(t, x):=\bar{P}(x)-P(t, x)$ with an adjusted $f_{h}$ according to $h$, we show

Theorem 1.2. For any given $\rho>0$ we get

$$
\max _{x \in \mathbb{R}}|\bar{P}(x)-P(t, x)|=N \frac{1}{\sqrt{t}} e^{-\rho t},
$$

where the constant $N$ depends only on $\rho$. Moreover, if $\rho \geq 1$, then we have

$$
\max _{x \in \mathbb{R}}|\bar{P}(x)-P(t, x)|=N e^{-\delta t} e^{-\rho t}, \quad t \geq 1,
$$

where the constants $N$ and $\delta>0$ depend only on $\rho$.

We prove Theorem 1.1 in Section 2 and Theorem 1.2 in Section 3.

\section{Asymptotic Behavior of the Free Boundary $\beta$ in $\Omega_{P}$}

The convexity of $\beta$ plays an important role in step 2 of the following proof.

Proof of Theorem 1.1. 1. We transform (1.7) into a form which we can analyze easily for our purpose. We set $\xi=\beta(s)$. We note that $\beta^{-1}$ exists since $\beta$ is strictly decreasing. Hence, we have

$$
G(t, \beta(t))=\rho \int_{\beta(t)}^{0} G\left(t-\beta^{-1}(\xi), \beta(t)-\xi\right) d \xi, \quad t>0 .
$$

Fix any $t_{0}>0$. Then for $t \in\left(t_{0}, \infty\right)$, implying $0>\beta\left(t_{0}\right)>\beta(t)$, we get

$$
G(t, \beta(t)) \geq \rho \int_{\beta(t)}^{\beta\left(t_{0}\right)} G\left(t-\beta^{-1}(\xi), \beta(t)-\xi\right) d \xi .
$$

Since $t-\beta^{-1}(\xi) \leq t-t_{0}$ for $\xi \in\left[\beta(t), \beta\left(t_{0}\right)\right]$, we get the following information for the integrand in (2.2):

$$
\begin{aligned}
G(t & \left.-\beta^{-1}(\xi), \beta(t)-\xi\right) \\
= & \frac{1}{\sqrt{4 \pi\left(t-\beta^{-1}(\xi)\right)}} \exp \left[-\frac{\left(\beta(t)-\xi+(\rho-1)\left(t-\beta^{-1}(\xi)\right)\right)^{2}}{4\left(t-\beta^{-1}(\xi)\right)}-\rho\left(t-\beta^{-1}(\xi)\right)\right] \\
& \geq \frac{1}{\sqrt{4 \pi\left(t-t_{0}\right)}} \exp \left[-\frac{\left(\beta(t)-\xi+(\rho-1)\left(t-\beta^{-1}(\xi)\right)\right)^{2}}{4\left(t-\beta^{-1}(\xi)\right)}-\rho\left(t-t_{0}\right)\right] .
\end{aligned}
$$

2. As an intermediate result, we will show

$$
\beta\left(t_{0}\right)-\bar{\beta} \leq \frac{1}{\rho} \exp \left[-\frac{\bar{\beta}(\rho-1)}{2}\right] \exp \left[-\frac{(\rho+1)^{2}}{4} t_{0}\right] .
$$

\footnotetext{
${ }^{1} \mathrm{~A}$ reviewer informed us of this.
} 
First, let us consider the case $0<\rho \leq 1$. The observation $\frac{\beta(t)-\beta\left(t_{0}\right)}{t-t_{0}} \leq \frac{\beta(t)-\xi}{t-\beta^{-1}(\xi)} \leq 0$ by the convexity of $\beta$ implies

$$
\begin{aligned}
-\frac{\left(\beta(t)-\xi+(\rho-1)\left(t-\beta^{-1}(\xi)\right)\right)^{2}}{4\left(t-\beta^{-1}(\xi)\right)} & =-\frac{1}{4}\left(\frac{\beta(t)-\xi}{t-\beta^{-1}(\xi)}+(\rho-1)\right)^{2}\left(t-\beta^{-1}(\xi)\right) \\
& =-\frac{1}{4}\left(-\frac{\beta(t)-\xi}{t-\beta^{-1}(\xi)}-(\rho-1)\right)^{2}\left(t-\beta^{-1}(\xi)\right) \\
& \geq-\frac{1}{4}\left(-\frac{\beta(t)-\beta\left(t_{0}\right)}{t-t_{0}}-(\rho-1)\right)^{2}\left(t-t_{0}\right) \\
& =-\frac{1}{4}\left(\frac{\beta(t)-\beta\left(t_{0}\right)}{t-t_{0}}+(\rho-1)\right)^{2}\left(t-t_{0}\right) \\
& =-\frac{\left(\beta(t)-\beta\left(t_{0}\right)+(\rho-1)\left(t-t_{0}\right)\right)^{2}}{4\left(t-t_{0}\right)},
\end{aligned}
$$

where the right hand side of (2.5) doesn't depend on $\xi \in\left[\beta(t), \beta\left(t_{0}\right)\right]$.

By (2.2), with the help of (2.3) and (2.5), we obtain

$$
\begin{aligned}
& \frac{1}{\sqrt{4 \pi t}} \exp \left[-\frac{(\beta(t)+(\rho-1) t)^{2}}{4 t}-\rho t\right] \\
& \geq \frac{\rho\left(\beta\left(t_{0}\right)-\beta(t)\right)}{\sqrt{4 \pi\left(t-t_{0}\right)}} \exp \left[-\frac{\left(\beta(t)-\beta\left(t_{0}\right)+(\rho-1)\left(t-t_{0}\right)\right)^{2}}{4\left(t-t_{0}\right)}-\rho\left(t-t_{0}\right)\right] .
\end{aligned}
$$

By obvious manipulations and simple calculations we have

$$
\begin{aligned}
& \frac{\sqrt{t-t_{0}}}{\sqrt{t}} \exp \left[-\frac{(\beta(t))^{2}}{4 t}+\frac{\left(\beta(t)-\beta\left(t_{0}\right)\right)^{2}}{4\left(t-t_{0}\right)}-\frac{t_{0}(\rho+1)^{2}}{4}-\frac{\beta\left(t_{0}\right)(\rho-1)}{2}\right] \\
\geq & \rho\left(\beta\left(t_{0}\right)-\beta(t)\right) .
\end{aligned}
$$

Since $\frac{\beta(t)}{t} \leq \frac{\beta(t)-\beta\left(t_{0}\right)}{t-t_{0}} \leq 0$ by the convexity of $\beta$ again, we further have

$$
\exp \left[-\frac{t_{0}(\rho+1)^{2}}{4}-\frac{\beta\left(t_{0}\right)(\rho-1)}{2}\right] \geq \rho\left(\beta\left(t_{0}\right)-\beta(t)\right) .
$$

Since $\beta(t)$ converges to $\bar{\beta}$ as $t \rightarrow \infty$ and $\bar{\beta} \leq \beta\left(t_{0}\right) \leq 0$, we get

$$
\exp \left[-\frac{\bar{\beta}(\rho-1)}{2}\right] \exp \left[-\frac{t_{0}}{4}(\rho+1)^{2}\right] \geq \rho\left(\beta\left(t_{0}\right)-\bar{\beta}\right)
$$

and (2.4).

The case $\rho>1$ is similar to the previous case. Using $\frac{\beta(t)-\beta\left(t_{0}\right)}{t-t_{0}} \leq \frac{\beta(t)-\xi}{t-\beta^{-1}(\xi)} \leq 0$ again, we observe

$$
\begin{aligned}
& -\frac{\left(\beta(t)-\xi+(\rho-1)\left(t-\beta^{-1}(\xi)\right)\right)^{2}}{4\left(t-\beta^{-1}(\xi)\right)} \\
= & -\frac{(\beta(t)-\xi)^{2}}{4\left(t-\beta^{-1}(\xi)\right)^{2}} \cdot\left(t-\beta^{-1}(\xi)\right)-\frac{1}{2}(\rho-1)(\beta(t)-\xi)-\frac{1}{4}(\rho-1)^{2}\left(t-\beta^{-1}(\xi)\right) \\
\geq & -\frac{\left(\beta(t)-\beta\left(t_{0}\right)\right)^{2}}{4\left(t-t_{0}\right)^{2}} \cdot\left(t-t_{0}\right)-\frac{1}{4}(\rho-1)^{2}\left(t-t_{0}\right)
\end{aligned}
$$


for $\xi \in\left[\beta(t), \beta\left(t_{0}\right)\right]$. If we proceed as before, we get

$$
\begin{aligned}
& \frac{1}{\sqrt{4 \pi t}} \exp \left[-\frac{(\beta(t)+(\rho-1) t)^{2}}{4 t}-\rho t\right] \\
& \geq \frac{\rho\left(\beta\left(t_{0}\right)-\beta(t)\right)}{\sqrt{4 \pi\left(t-t_{0}\right)}} \exp \left[-\frac{\left[\beta(t)-\beta\left(t_{0}\right)\right]^{2}}{4\left(t-t_{0}\right)}-\frac{1}{4}(\rho-1)^{2}\left(t-t_{0}\right)-\rho\left(t-t_{0}\right)\right]
\end{aligned}
$$

and, using $\frac{\beta(t)}{t} \leq \frac{\beta(t)-\beta\left(t_{0}\right)}{t-t_{0}} \leq 0$, we obtain

$$
\begin{aligned}
& \exp \left[-\frac{t_{0}(\rho+1)^{2}}{4}-\frac{\beta(t)(\rho-1)}{2}\right] \\
& \geq \frac{\sqrt{t-t_{0}}}{\sqrt{t}} \exp \left[-\frac{(\beta(t))^{2}}{4 t}+\frac{\left(\beta(t)-\beta\left(t_{0}\right)\right)^{2}}{4\left(t-t_{0}\right)}-\frac{t_{0}(\rho+1)^{2}}{4}-\frac{\beta(t)(\rho-1)}{2}\right] \\
& \geq \rho\left(\beta\left(t_{0}\right)-\beta(t)\right) .
\end{aligned}
$$

Letting $t \rightarrow \infty$, we obtain (2.4). We denote $N_{1}=N_{1}(\rho)=\frac{1}{\rho} \exp \left[-\frac{\bar{\beta}(\rho-1)}{2}\right]=$ $\frac{1}{\rho}\left(1+\frac{1}{\rho}\right)^{\frac{\rho-1}{2}}$.

3. As we consider (2.1), we have

$$
\begin{aligned}
& \frac{1}{\sqrt{4 \pi t}} \exp \left[-\frac{(\beta(t)+(\rho-1) t)^{2}}{4 t}-\rho t\right] \\
= & \rho \int_{\beta(t)}^{0} \frac{1}{\sqrt{4 \pi\left(t-\beta^{-1}(\xi)\right)}} \exp \left[-\frac{\left(\beta(t)-\xi+(\rho-1)\left(t-\beta^{-1}(\xi)\right)\right)^{2}}{4\left(t-\beta^{-1}(\xi)\right)}\right. \\
& \left.-\rho\left(t-\beta^{-1}(\xi)\right)\right] d \xi
\end{aligned}
$$

for $t>0$. A manipulation and calculations show

$$
\begin{aligned}
\frac{1}{\rho}=\int_{\beta(t)}^{0} \frac{1}{\sqrt{1-\frac{\beta^{-1}(\xi)}{t}}} \exp \left[\frac{\xi(\rho-1)}{2}+\frac{(\rho+1)^{2}}{4} \beta^{-1}(\xi)\right. \\
\left.\quad+\frac{(\beta(t))^{2}}{4 t}-\frac{(\beta(t)-\xi)^{2}}{4\left(t-\beta^{-1}(\xi)\right)}\right] d \xi \\
=\int_{\bar{\beta}}^{0} I_{(\beta(t), 0)}(\xi) \frac{1}{\sqrt{1-\frac{\beta^{-1}(\xi)}{t}}} \cdot g(\rho, t, \xi) d \xi
\end{aligned}
$$

where

$$
g(\rho, t, \xi):=\exp \left[\frac{\xi(\rho-1)}{2}+\frac{(\rho+1)^{2}}{4} \beta^{-1}(\xi)+\frac{(\beta(t))^{2}}{4 t}-\frac{(\beta(t)-\xi)^{2}}{4\left(t-\beta^{-1}(\xi)\right)}\right]
$$

and $I_{(\beta(t), 0)}(\xi)$ is the indicator function on the interval $(\beta(t), 0)$. We note that for any fixed $\xi \in(\bar{\beta}, 0)$, there exists $t(\xi)$ such that $\beta(t)<\xi$ (hence, $t>\beta^{-1}(\xi)$ ) for $t>t(\xi)$. For such a $t$, we have $I_{(\beta(t), 0)}(\xi)=1$ and

$$
\lim _{t \rightarrow \infty} \frac{\beta^{-1}(\xi)}{t} \rightarrow 0, \quad \lim _{t \rightarrow \infty} g(\rho, t, \xi)=\exp \left[\frac{\xi(\rho-1)}{2}+\frac{(\rho+1)^{2}}{4} \beta^{-1}(\xi)\right] .
$$


By Fatou's lemma, we obtain

$$
\begin{aligned}
\frac{1}{\rho} & \geq \liminf _{t \rightarrow \infty} \int_{\bar{\beta}}^{0} I_{(\beta(t), 0)}(\xi) \frac{1}{\sqrt{1-\frac{\beta^{-1}(\xi)}{t}}} \cdot g(\rho, t, \xi) d \xi \\
& \geq \int_{\bar{\beta}}^{0} \liminf _{t \rightarrow \infty}\left[I_{(\beta(t), 0)}(\xi) \frac{1}{\sqrt{1-\frac{\beta^{-1}(\xi)}{t}}} \cdot g(\rho, t, \xi)\right] d \xi \\
& =\int_{\bar{\beta}}^{0} \exp \left[\frac{(\rho-1)}{2} \xi+\frac{(\rho+1)^{2}}{4} \beta^{-1}(\xi)\right] d \xi .
\end{aligned}
$$

Our change of variable $\beta(t)=\xi$ gives us

$$
\begin{aligned}
\frac{1}{\rho} & \geq \int_{0}^{\infty} \exp \left[\frac{(\rho-1)}{2} \beta(s)+\frac{(\rho+1)^{2}}{4} s\right]\left(-\beta^{\prime}(s)\right) d s \\
& =\int_{0}^{\infty}(\bar{\beta}-\beta(s))^{\prime} \exp \left[\frac{\rho-1}{2} \beta(s)+\frac{(\rho+1)^{2}}{4} s\right] d s
\end{aligned}
$$

If $\rho>1$, we have

$$
\frac{1}{\rho} \geq e^{\frac{\rho-1}{2} \bar{\beta}} \int_{0}^{\infty}(\bar{\beta}-\beta(s))^{\prime} e^{\frac{(\rho+1)^{2}}{4} s} d s \geq e^{\frac{\rho-1}{2} \bar{\beta}} \int_{0}^{t_{0}}(\bar{\beta}-\beta(s))^{\prime} e^{\frac{(\rho+1)^{2}}{4} s} d s
$$

for any $t_{0}$ since $\bar{\beta}<\beta(s)<0$. By integration by parts, we have

$$
\begin{aligned}
& \int_{0}^{t_{0}}(\bar{\beta}-\beta(s))^{\prime} e^{\frac{(\rho+1)^{2}}{4} s} d s \\
& =\left[(\bar{\beta}-\beta(s)) e^{\frac{(\rho+1)^{2}}{4} s}\right]_{0}^{t_{0}}-\frac{(\rho+1)^{2}}{4} \int_{0}^{t_{0}}(\bar{\beta}-\beta(s)) e^{\frac{(\rho+1)^{2}}{4} s} d s \\
& =\left(\bar{\beta}-\beta\left(t_{0}\right)\right) e^{\frac{(\rho+1)^{2}}{4} t_{0}}-\bar{\beta}+\frac{(\rho+1)^{2}}{4} \int_{0}^{t_{0}}(\beta(s)-\bar{\beta}) e^{\frac{(\rho+1)^{2}}{4} s} d s .
\end{aligned}
$$

This implies

$$
\frac{1}{\rho} e^{-\frac{\rho-1}{2} \bar{\beta}} \geq\left(\bar{\beta}-\beta\left(t_{0}\right)\right) e^{\frac{(\rho+1)^{2}}{4} t_{0}}-\bar{\beta}+\frac{(\rho+1)^{2}}{4} \int_{0}^{t_{0}}(\beta(s)-\bar{\beta}) e^{\frac{(\rho+1)^{2}}{4} s} d s
$$

for any $t_{0} \geq 0$. By letting $t_{0} \rightarrow \infty$ in (2.7) and using the Monotone Convergence Theorem, we get

$$
\frac{1}{\rho} e^{-\frac{\rho-1}{2} \bar{\beta}} \geq \liminf _{t_{0} \rightarrow \infty}\left(\left(\bar{\beta}-\beta\left(t_{0}\right)\right) e^{\frac{(\rho+1)^{2}}{4} t_{0}}\right)-\bar{\beta}+\frac{(\rho+1)^{2}}{4} \int_{0}^{\infty}(\beta(s)-\bar{\beta}) e^{\frac{(\rho+1)^{2}}{4} s} d s .
$$

We note that $\left|\left(\bar{\beta}-\beta\left(t_{0}\right)\right) e^{\frac{(\rho+1)^{2}}{4} t_{0}}\right| \leq N_{1}(\rho)$ by (2.4). Hence, we obtain

$$
\int_{0}^{\infty}(\beta(s)-\bar{\beta}) e^{\frac{(\rho+1)^{2}}{4} s} d s<\infty
$$

which implies the claim of the theorem.

In the case $\rho \leq 1$ we get (2.8) similarly. The only difference is that we start with

$$
\frac{1}{\rho} \geq \int_{0}^{\infty}(\bar{\beta}-\beta(s))^{\prime} e^{\frac{(\rho+1)^{2}}{4} s} d s \geq \int_{0}^{t_{0}}(\bar{\beta}-\beta(s))^{\prime} e^{\frac{(\rho+1)^{2}}{4} s} d s, \quad t_{0}>0
$$

instead of (2.6). The theorem is proved. 
Remark 2.1. We note that if the volatility gets smaller, that is, $\sigma \rightarrow 0^{+}(\rho \rightarrow \infty)$, then $\bar{\beta} \rightarrow 0$, and $N_{1}(\rho) \rightarrow 0$ by the observation that $\left(1+\frac{1}{\rho}\right)^{\rho} \rightarrow e$.

Remark 2.2. Going back to $b$, one can easily derive

$$
\ln \left(\frac{b(\tau)}{\bar{b}}\right)=o\left(\exp \left[-\frac{(\rho+1)^{2} \sigma^{2}}{8} \tau\right]\right)
$$

as $\tau \rightarrow \infty$.

\section{Asymptotic behavior of the Rescaled price $P$ of American put}

The rescaled perpetual option price $\bar{P}=\bar{P}(x)$ defined in (1.6) is the solution of the following stationary version of (1.4), (1.5):

$$
-\bar{P}_{x x}-(\rho-1) \bar{P}_{x}+\rho \bar{P}=0 \quad \text { for } \quad x>\bar{\beta}, \quad \bar{P}(x)=1-e^{x} \quad \text { for } \quad x \leq \bar{\beta}
$$

with conditions $\bar{P}_{x}(\bar{\beta})=-e^{\bar{\beta}}, \lim _{x \rightarrow \infty} \bar{P}(x)=0$. The difference function $h(t, x):=$ $\bar{P}(x)-P(t, x)$ is positive since $P(t, x) \nearrow \bar{P}(x)$ as $t \rightarrow \infty$ and, by (1.4) with conditions (1.5), (3.1) with attached conditions, $h$ can be expressed in the following way:

$$
h(t, x)= \begin{cases}\frac{1}{1+\rho} e^{-\rho(x-\bar{\beta})}-P(t, x) & \text { if } t \geq 0, x>\beta(t) \\ \frac{1}{1+\rho} e^{-\rho(x-\bar{\beta})}-1+e^{x} & \text { if } t \geq 0, \bar{\beta}<x \leq \beta(t), \\ 0 & \text { if } t \geq 0, x \leq \bar{\beta}\end{cases}
$$

and $h$ satisfies

$$
h_{t}-h_{x x}-(\rho-1) h_{x}+\rho h= \begin{cases}0 & \text { if } t>0, x>\beta(t) \\ -\rho & \text { if } t>0, \bar{\beta}<x \leq \beta(t), \\ 0 & \text { if } t>0, x \leq \bar{\beta}\end{cases}
$$

with conditions

$$
h_{x}(t, \beta(t))=-\frac{\rho}{\rho+1} e^{-\rho(\beta(t)-\bar{\beta})}+e^{\beta(t)}, \quad \lim _{x \rightarrow \infty} h(t, x)=0 .
$$

The following extra information of $h$ on the boundary is available.

Lemma 3.1. We have

$$
h_{x}(t, \beta(t))>0, \quad \frac{d}{d t}(h(t, \beta(t)))<0, \quad t>0 .
$$

Proof. 1. The following are equivalent and true:

$$
\begin{aligned}
& \beta(t)>\bar{\beta}=\ln \left(\frac{\rho}{\rho+1}\right), e^{\beta(t)}>\frac{\rho}{\rho+1}, e^{(\rho+1) \beta(t)}>\left(\frac{\rho}{\rho+1}\right)^{\rho+1}, \\
& e^{\beta(t)}-\left(\frac{\rho}{\rho+1}\right)^{\rho+1} e^{-\rho \beta(t)}>0 .
\end{aligned}
$$

By the first condition in (3.4), the right hand side of (3.5) is just $h_{x}(t, \beta(t))$. The first claim is proved.

2. Since $\frac{d}{d t}(h(t, \beta(t)))=h_{t}(t, \beta(t))+h_{x}(t, \beta(t)) \beta^{\prime}(t)$, the second claim also follows by the observation $h_{t}(t, \beta(t))=P_{t}(t, \beta(t))=0$ (see (1.11)) and the first assertion. 
At first, the Maximum Principle (see, for instance, [6] or [9]) seems a good tool for estimating the behavior of $h$. But, if we apply the Maximum Principle directly, we get

$$
\max _{x \geq \beta(t)} h(t, x) \leq \max \left\{e^{-\rho\left(t-t_{0}\right)} \max _{x \geq \beta\left(t_{0}\right)} h\left(t_{0}, x\right), \max _{t_{0} \leq s<t} h(s, \beta(s))\right\}, \quad t>t_{0} .
$$

Since Lemma 3.1 implies

$$
\max _{t_{0} \leq s<t} h(s, \beta(s))=h\left(t_{0}, \beta\left(t_{0}\right)\right),
$$

in the case $t_{0}=0$, we receive

$$
\begin{aligned}
\max _{x \geq \beta(t)} h(t, x) & \leq \max \left\{e^{-\rho t} \max _{x \geq 0} h(0, x), h(0,0)\right\} \\
& =h(0,0) \cdot \max \left\{e^{-\rho t}, 1\right\}=\frac{1}{\rho+1}\left(\frac{\rho}{\rho+1}\right)^{\rho}, \quad t>0
\end{aligned}
$$

by (3.2). As we see, we have obtained mere boundedness of $h$.

Remark 3.2. Using a comparison argument, the Maximum Principle, and Theorem 1.1, one can prove the following statement, which is better than boundedness but weaker than Theorem 1.2 ,

For any given $\rho>0$ it follows that

$$
\max _{x \in \mathbb{R}}|\bar{P}(x)-P(t, x)| \leq N_{1}(\rho) e^{-\rho t}, \quad t \geq 0,
$$

where the constant $N_{1}(\rho)$ comes from the end of step 2 in the proof of Theorem 1.1 .

From this one can derive the long time asymptotic behavior of the option price $F$ given by

$$
\max _{S>0}|\bar{F}(S)-F(\tau, S)| \leq K N_{2}(\rho) e^{-r \tau}, \quad \tau \geq 0 .
$$

As in Remark 2.1 we have $N_{2}(\rho)=N_{1}(\rho) \rightarrow 0^{+}$as $\rho \rightarrow \infty$.

Remark 3.3. If we disregard $N_{2}(\rho)$ in (3.6), we can easily obtain (3.6) with 1 in place of $N_{2}(\rho)$ from the probabilistic definition of the option price 2 in the following way.

For any given $\tau, S$ the option price $F(\tau, S)$ is defined by using stopping times with respect to the filtration generated by the process $S$. Starting at $S$ :

$$
F(\tau, S)=\sup _{\mathcal{T} \in \mathfrak{T}_{\tau}} \mathbb{E} e^{-r \mathcal{T}}\left(K-S_{\mathcal{T}}\right)_{+}
$$

where $\mathfrak{T}_{\tau}$ is the collection of all stopping times less than or equal to $\tau$. For any $\tau_{1} \geq \tau$, we have

$$
F\left(\tau_{1}, S\right)=\max \left\{\sup _{\mathcal{T} \in \mathfrak{T}_{\tau}} \mathbb{E} e^{-r \mathcal{T}}\left(K-S_{\mathcal{T}}\right)_{+}, \sup _{\mathcal{T} \in \mathfrak{T}_{\tau_{1} \backslash \mathfrak{T}_{\tau}}} \mathbb{E} e^{-r \mathcal{T}}\left(K-S_{\mathcal{T}}\right)_{+}\right\} .
$$

We notice that $\sup _{\mathcal{T} \in \mathfrak{T}_{\tau}} \mathbb{E} e^{-r \mathcal{T}}\left(K-S_{\mathcal{T}}\right)_{+}=F\left(\tau_{1}, S\right)$. On the other hand, for any $\mathcal{T} \in \mathfrak{T}_{\tau_{1}} \backslash \mathfrak{T}_{\tau}$, we have

$$
\begin{aligned}
\mathbb{E} e^{-r \mathcal{T}}\left(K-S_{\mathcal{T}}\right)_{+} & \leq \mathbb{E} I_{\mathcal{T} \leq \tau} e^{-r \mathcal{T}}\left(K-S_{\mathcal{T}}\right)_{+}+\mathbb{E} I_{\mathcal{T}>\tau} e^{-r \mathcal{T}}\left(K-S_{\mathcal{T}}\right)_{+} \\
& \leq F(\tau, S)+K e^{-r \tau}
\end{aligned}
$$

${ }^{2}$ This was pointed out by a reviewer. We appreciate it. 
Hence, we have $F\left(\tau_{1}, S\right)-F(\tau, S) \leq K e^{-r \tau}$, and by letting $\tau_{1} \rightarrow \infty$ and considering all $S>0$, we get

$$
\max _{S>0}|\bar{F}(S)-F(\tau, S)| \leq K e^{-r \tau}
$$

For the proof of Theorem 1.2 we use the solution representation of (3.3) in the form of (1.8). By (3.2) the representation can be specified by the following:

$h(t, x)$

$$
=\int_{0}^{\infty} G(t, x-y) \frac{1}{1+\rho} e^{-\rho(y-\bar{\beta})} d y+\int_{\bar{\beta}}^{0} G(t, x-y)\left[\frac{1}{1+\rho} e^{-\rho(y-\bar{\beta})}-1+e^{y}\right] d y
$$

$$
-\rho \int_{0}^{t} \int_{\bar{\beta}}^{\beta(s)} G(t-s, x-y) d s .
$$

Proof of Theorem 1.2. 1. The equation (3.7) is

$$
\begin{aligned}
h(t, x)= & \int_{\bar{\beta}}^{\infty} G(t, x-y) \frac{1}{1+\rho} e^{-\rho(y-\bar{\beta})} d y+\int_{\bar{\beta}}^{0} G(t, x-y)\left(-1+e^{y}\right) d y \\
& -\rho \int_{0}^{t} \int_{\bar{\beta}}^{\beta(s)} G(t-s, x-y) d s,
\end{aligned}
$$

where the second and third terms on the right hand side are negative. We thus have

$$
\begin{aligned}
h(t, x) & \leq \int_{\bar{\beta}}^{\infty} G(t, x-y) \frac{1}{1+\rho} e^{-\rho(y-\bar{\beta})} d y \\
& \leq \frac{1}{\rho+1} \int_{\bar{\beta}}^{\infty} G(t, x-y) d y \\
& =\frac{1}{\rho+1} \int_{\bar{\beta}}^{\infty} \frac{1}{\sqrt{4 \pi t}} \exp \left[-\frac{(x-y+(\rho-1) t)^{2}}{4 t}-\rho(y-\bar{\beta})\right] d y \cdot e^{-\rho t} .
\end{aligned}
$$

Note that we need to consider only $x \geq \bar{\beta}$. By a change of variables the integral part of (3.8) is expressed by

$$
\alpha(t, z):=\int_{0}^{\infty} \frac{1}{\sqrt{4 \pi t}} \exp \left[-\frac{(z+b t-y)^{2}}{4 t}-(b+1) y\right] d y
$$

with $b=\rho-1$ and $z=x-\bar{\beta} \geq 0$.

2. For any $\rho>0$ we get

$$
\sup _{z \geq 0}|\alpha(t, z)| \leq N \frac{1}{\sqrt{t}}
$$

for some constant $N$ depending only on $\rho$. In fact, we have

$$
|\alpha(t, z)| \leq \frac{1}{\sqrt{4 \pi t}} \int_{0}^{\infty} e^{-\rho y} d y=\frac{1}{\sqrt{4 \pi t}} \cdot \frac{1}{\rho} .
$$

This and (3.8) deal with the first assertion of the theorem. 
3. Now, we assume that $b \geq 0$, i.e., $\rho \geq 1$. By changes of variables, we observe

$$
\begin{aligned}
& \alpha(t, z) \\
&= \int_{-(z+b t)}^{\infty} \frac{1}{\sqrt{4 \pi t}} \exp \left[-\frac{y^{2}}{4 t}-(b+1) y\right] d y \cdot \exp [-(b+1) z-b(b+1) t] \\
&= \frac{1}{\sqrt{\pi}} \int_{-\frac{z+b t}{\sqrt{4 t}}}^{\infty} \exp \left[-\eta^{2}-2(b+1) \sqrt{t} \eta\right] d \eta \cdot \exp [-(b+1) z-b(b+1) t] \\
&= \frac{1}{\sqrt{\pi}} \int_{-\frac{z+b t}{\sqrt{4 t}}}^{\infty} \exp \left[-(\eta+(b+1) \sqrt{t})^{2}\right] d \eta \cdot \exp \left[-(b+1) z-b(b+1) t+(b+1)^{2} t\right] \\
&= \frac{1}{\sqrt{\pi}} \int_{-\frac{z+b t}{\sqrt{4 t}}+(b+1) \sqrt{t}}^{\infty} e^{-\xi^{2}} d \xi \cdot \exp \left[-(b+1) z-b(b+1) t+(b+1)^{2} t\right] \\
&= \frac{1}{\sqrt{\pi}} \int_{\frac{-z+(b+2) t}{\sqrt{4 t}}}^{\infty} e^{-\xi^{2}} d \xi \cdot \exp [-(b+1) z+(b+1) t] . \\
& \text { If }-z+(b+2) t \leq \frac{1}{2}(b+2) t, \text { i.e., } z \geq \frac{1}{2}(b+2) t \text { or } x \geq \bar{\beta}+\frac{1}{2}(b+2) t, \text { then we get } \\
& \quad \alpha(t, z) \leq \frac{1}{\sqrt{\pi}} \int_{-\infty}^{\infty} e^{-\xi^{2}} d \xi \cdot \exp \left[-\frac{1}{2}(b+1)(b+2) t+(b+1) t\right] \\
& \quad=1 \cdot \exp \left[-\frac{1}{2} b(b+1) t\right]
\end{aligned}
$$

which implies

$$
h(t, x) \leq \frac{1}{\rho+1} \cdot e^{-\frac{1}{2}(\rho-1) \rho t} \cdot e^{-\rho t} .
$$

If $-z+(b+2) t>\frac{1}{2}(b+2) t$, i.e., $(0 \leq) z<\frac{1}{2}(b+2) t$ or $\bar{\beta} \leq x<\bar{\beta}+\frac{1}{2}(b+2) t$, then, using the inequality $\int_{a}^{\infty} e^{-u^{2}} d u \leq \frac{1}{2 a} e^{-a^{2}}$ for $a>0$, we have

$$
\begin{aligned}
\alpha(t, z) & \leq \frac{1}{\sqrt{\pi}} \frac{2 \sqrt{t}}{2(-z+(b+2) t)} \exp \left[-\frac{(-z+(b+2) t)^{2}}{4 t}-(b+1) z+(b+1) t\right] \\
& \leq \frac{1}{\sqrt{\pi}} \frac{2}{(b+2) \sqrt{t}} \exp \left[-\frac{z^{2}}{4 t}-\frac{b}{2} z-\frac{b^{2}}{4} t\right] \\
& \leq \frac{1}{\sqrt{\pi}} \frac{2}{\rho+1} \frac{1}{\sqrt{t}} e^{-\frac{(\rho-1)^{2}}{4} t}
\end{aligned}
$$

where we used the assumption $b \geq 0$ for the last inequality. It follows that

$$
h(t, x) \leq \frac{1}{\sqrt{\pi}} \frac{2}{(\rho+1)^{2}} \cdot \frac{1}{\sqrt{t}} e^{-\frac{(\rho-1)^{2}}{4} t} \cdot e^{-\rho t} .
$$

We proved the second assertion of the theorem; we can take $\delta=\frac{(\rho-1)^{2}}{4}$. The theorem follows.

\section{ACKNOWLEDGEMENT}

The authors would like to express sincere thanks for the helpful information and advice from Professor Hyeng Keun Koo at Ajou University. 


\section{REFERENCES}

1. Xinfu Chen; John Chadam, A mathematical analysis of the optimal exercise boundary for American put options, Siam J. Math. Anal. 38, No. 5 (2006/07), 1613-1641. MR 2286022 (2007k:91131)

2. Xinfu Chen; John Chadam; Lishang Jiang; Weian Zheng, Convexity of the exercise boundary of the American put option on a zero dividend asset, Math. Finance 18, No. 1 (2008), 185-197. MR 2380946(2008m:91109)

3. Erik Ekström, Convexity of the optimal stopping boundary for the American put option, J. Math. Anal. Appl. 299, No. 1 (2004), 147-156. MR2091277 (2005f:91068)

4. Jonathan Goodman; Daniel N. Ostrov, On the early exercise boundary of the American put option, Siam J. Appl. Math. 62, No. 5 (2002), 1823-1835. MR1918579 (2003i:35120)

5. John C. Hull, Options, Futures, and Other Derivatives. 5th edition, Prentice Hall, NJ, 2002.

6. A.M. Il'in; A.S. Kalashnikov; O.A. Oleŭnik, Second-order linear equations of parabolic type, J. Math. Sciences (New York) 108, No. 4 (2002), 435-542. MR.1875963 (2003a:35087)

7. S. D. Jacka, Optimal stopping and the American put, Math. Finance 1, No. 2 (1991), 1-14.

8. Robert C. Merton, Continuous-time finance, B. Blackwell, Cambridge, MA, 1990.

9. R. M. Redheffer; W. Walter, The total variation of solutions of parabolic differential equations and a maximum principle in unbounded domains, Math. Ann. 209 (1974), 57-67. MR0344683 (49:9422)

10. Steven E. Shreve, Stochastic calculus for finance. II. Continuous-time models, SpringerVerlag, New York, 2004. MR.2057928 (2005c:91001)

11. P. Wilmott; J. Dewynne; S. Howison, Option Pricing: Mathematical Models and Computation, Cambridge University Press, New York, 1995.

Department of Mathematics, Yonsei University, Seoul 120-749 Korea

E-mail address: purehope@yonsei.ac.kr

Department of Mathematics, Yonsei University, Seoul 120-749 Korea

E-mail address: choe@yonsei.ac.kr

Department of Mathematics, Ajou University, Suwon 443-749 Korea

E-mail address: kijung@ajou.ac.kr 